\title{
Existence and computation of optimally localized coherent states
}

\author{
Matthias Holschneider* Gerd Teschke ${ }^{\dagger}$
}

27th September 2005

\begin{abstract}
This paper is concerned with localization properties of coherent states. Instead of classical uncertainty relations we consider 'generalized' localization quantities. This is done by introducing measures on the reproducing kernel. In this context we may prove the existence of optimally localized states. Moreover, we provide a numerical scheme for deriving them.
\end{abstract}

\section{Motivation}

The goal of this paper is to introduce a new concept of localization. In classical lore, one typically considers uncertainty relations. In this framework it is well known that a nonzero state or wavefunction cannot be arbitrarily well localized simultaneously in space and Fourier domain. This fact may be quantified by the Heisenberg uncertainty relation,

$$
\Delta x \Delta k \geq 2 \pi
$$

There are functions which are optimally localized in phase space in that they satisfy the inequality with the lower bound. For a detailed analysis on uncertainty relations in the context of Gabor and wavelet transforms we refer, e.g, to [11, 12, 5, 18].

However, for certain physical applications, e.g. radar imaging, it is very promizing to ask for more flexible concepts. In principle, in radar imaging one has to evaluate the output of the correlation receiver, see [1]. An important role in detecting objects is played by the radar ambiguity function, which is ideally given by a delta function. In the narrowband regime, which is of interest in radar imaging, the ambiguity function is given by $\Pi(\phi, \tau)=\left\langle\Psi_{\phi, \tau}, \Psi_{\phi_{0}, \tau_{0}}\right\rangle$, where $\Psi$ stands for the transmitted waveform. Since a delta pulse is obviously not possible to realize, one currently uses pulses that minimize by means of its band/doppler-width and its pulse/range-width the so-called narrowband (Heisenberg) uncertainty principle, see $[1,7,6,4]$. A minimization of the Heisenberg quantities leads

*Universität Potsdam, Institut fr Mathematik, Postfach 6015 53, 14415 Potsdam, Germany.

$\dagger$ Universität Bremen, Zentrum für Technomathematik, Postfach 3304 40, 28334 Bremen, Germany. G. T. was partially supported by Deutsche Forschungsgemeinschaft Grants TE 354/1-2, TE 354/3-1 
to pulses with Gaussian envelope function. But this is difficult to implement too and, moreover, allows no flexibility in specifying more sophisticated localization attributes; e.g., in order to obtain an accurate spatial resolution while allowing a certain frequency uncertainty, the ambiguity function $\Pi$ should have fast decay with respect to range and a moderate decay with respect to doppler frequency, e.g.

$$
|\Pi(\phi, \tau)| \leq(1+|\tau|)^{-4}(1+\log (1+|\phi|))^{-1} .
$$

With the theory presented here in the paper at hand, we may now proceed as follows: given the specific localization characteristic (1.1) in the range doppler plane, compute the optimal waveform to be transmitted. To be more general, we consider measures of uncertainty or delocalization in phase space (range doppler plane) and we shall prove the existence of optimally localized states (waveforms).

In principle, general wavelet transforms

$$
s \mapsto \mathcal{W}_{g} s, \quad \mathcal{W}_{g} s(x)=(U(x) g, s)_{H}, \quad x \in G
$$

associated with the square integrable irreducible representation $U$ of a locally compact group $G$ provides a one to one correspondence between the state Hilbert space $H$ and a reproducing kernel Hilbert space over the group. The reproducing kernel is up to a normalization the wavelet transform of the wavelet itself

$$
\Pi=\mathcal{W}_{g} g
$$

This kernel can be interpreted as the Heisenberg box of the phase space. In this paper we shall be concerned with localization properties of these reproducing kernels. In fact the reproducing kernel of wavelet analysis cannot be arbitrarily well localized. For instance, there is no wavelet such that the associated reproducing kernel is compactly supported. Obviously here is no universal way of quantifying localization. Instead we propose to quantify localization through the following and similar families of cost functionals

$$
\Phi[g]=\sup _{x \in G} w(x)^{-1}\left|\mathcal{W}_{g} g(x)\right|
$$

where $w$ is some positive weight, decaying "at $\infty$ ". We will prove that this functional and similar "localization" functionals are weakly lower semi-continuous. It therefore has a minimizer over any weak* compact set. In other words: for any such measure of localization there is at least one optimal state.

Localization of wavelet transforms has been considered before, see e.g. [17, 15, 9, 8]. In $[9,8]$ the authors consider localizing wavelet coefficients with respect to some preassigned analyzing wavelet and a compact subset in the wavelet plane. In their approach the analyzing wavelet was fixed. In this paper, however, we consider the nonlinear problem of optimizing the localization of the reproducing kernel. Since the reproducing kernel depends quadratically on the underlying wavelet, this problem is by nature highly nonlinear and therefore an explicit expression of the optimal state seems to remain a pipe dream. However, in the last section we discuss a numerical procedure to approximately compute such optimally localized states. 


\section{The basic formulas}

Let us recall the basic formulas of continuous wavelet transform associated with a square integrable group representation. We only recall the few facts that are necessary for this paper. For more details we refer to, e.g., [10], [13]. Let $G$ be a non-compact, locally compact, and $\sigma$ compact topological group and $G \ni g \mapsto U(g)$ a unitary, strongly continuous, irreducible, square integrable representation in some Hilbert space $H$. The wavelet transform of $s \in H$ with respect to $g \in H$ is point-wise defined for $x \in G$

$$
\mathcal{W}_{g} s(x)=(U(x) g, s)_{H}
$$

The left and the right invariant Haar measures are denoted by $d \lambda$ and $d \rho$. They are defined up to some positive factor. Over $G$ we consider the two Hilbert spaces $L^{2}(G, d \lambda)$ and $L^{2}(G, d \rho)$. We suppose that $d \lambda$ and $d \rho$ are scaled suitably so that the mapping $s(x) \mapsto s\left(x^{-1}\right)$ is an isometry between these two Hilbert spaces. A wavelet is called admissible, if $\mathcal{W}_{g} g \in L^{2}(G, d \lambda)$. Thanks to the formula

$$
\mathcal{W}_{g} s(x)=\mathcal{W}_{s} g\left(x^{-1}\right)
$$

admissibility is also equivalent to $\mathcal{W}_{g} g \in L^{2}(G, d \rho)$. We denote the set of all admissible wavelets by $A$. For $g, h \in A$ and $s, u \in H$ the following equation holds

$$
\left(\mathcal{W}_{g} s, \mathcal{W}_{h} u\right)_{L^{2}(G, d \lambda)}=C(g, h)(s, u)_{H},
$$

where $C$ is a densely defined, closed, positive quadratic form. Its form domain is precisely $A$. We write $c_{g, h}=C(g, h)$ and $c_{g}=C(g, g)$. By the first representation theorem there is a closed, linear operator $T$ such that for all $u \in D(T)$ and $v \in A$ we have $C(v, u)=(v, T u)_{H}$. The space $A$ is in general a non-closed subspace of $H$. However, since $C$ is a closed quadratic form it becomes a Hilbert space with respect to

$$
(s, u)_{A}=(s, u)_{H}+C(s, r), \quad\|s\|_{A}^{2}=\|s\|_{H}^{2}+c_{s} .
$$

Convergence in $A$ will be understood with respect to this norm. From equation (2.1) it follows that in particular $\mathcal{W}_{g}$ is for admissible, non-zero $g$ a multiple of an isometry

$$
\left\|\mathcal{W}_{g} s\right\|_{L^{2}(G, d \lambda)}^{2}=c_{g}\|s\|_{H}^{2} .
$$

The adjoint of the wavelet analysis is a wavelet synthesis

$$
\mathcal{W}_{g}^{*}=\mathcal{M}_{g}
$$

Formally it can be written as follows

$$
\mathcal{M}_{h} r(x)=\int_{G} r(x) U(x) h d \lambda(x) .
$$

We have for $g, h \in A$

$$
\mathcal{M}_{h} \mathcal{W}_{g}=c_{g, h} 1
$$


The combination $\mathcal{W}_{g} \mathcal{M}_{h}$ can be written as non-commutative convolution operator. If we define on $L^{2}(G, d \lambda) \times L^{2}(G, d \lambda)$ the (left) convolution product as

$$
\Pi * r(x)=\int_{G} \Pi\left(y^{-1} \circ x\right) r(y) d \lambda(y),
$$

we have for $g, h \in A$

$$
\mathcal{W}_{g} \mathcal{M}_{h}=\Pi *, \quad \Pi=\mathcal{W}_{g} h .
$$

In particular we will use the following formula over and over

$$
\mathcal{W}_{h}=\Pi * \mathcal{W}_{g}, \quad \Pi=c_{g, h}^{-1} \mathcal{W}_{h} h
$$

\section{General Localizations}

Before formulating the general existence theorem we consider the particular case of localization measures through a weighted $L^{\infty}$ norm. Consider therefore a positive function $w: G \rightarrow R_{+}$. We suppose that $w$ is symmetric

$$
w\left(x^{-1}\right)=w(x) .
$$

and invariant under the $G$ action in that for all $y \in G$ we can find a $c>0$ such that

$$
w(y \circ x) \leq c w(x), \quad w(x \circ y) \leq c w(x) .
$$

Then $w \in L^{2}(G, d \lambda)$ is equivalent with $w \in L^{2}(G, d \rho)$. Moreover, we consider weights, for which either of both (and hence both) of the following holds

$$
w * w \in L^{2}(G, d \lambda), \quad w * w \in L^{2}(G, d \rho) .
$$

A natural measure for the localization of a function $r$ over $G$ with respect to $w$ is the following weighted norm

$$
\sup _{G} w^{-1}|r| .
$$

For fixed $h \in A$ let $\Sigma_{h} \subset H$ denote the affine subspace of co-dimension 1 defined through $\Sigma_{h}=\left\{g \in H:(g, T h)_{H}=1\right\}$. Note that $\Sigma_{h}$ may contain non-admissible vectors. The admissible wavelets in $\Sigma_{h}$ are reconstruction wavelets for $h$ :

$$
\mathcal{M}_{h} \mathcal{W}_{g}=\mathcal{M}_{g} \mathcal{W}_{h}=1
$$

We introduce the following functional on $H$.

$$
\Phi[s]=\Phi_{w}[s]=\sup _{G} w^{-1}\left|\mathcal{W}_{s} s\right|,
$$

whenever the right hand side is finite. In all other cases we set $\Phi[s]=\infty$. We now can formulate the first theorem. 
Theorem 1 Let $h \in H, h \neq 0$, be such that $\Phi[h]<\infty$. Then there exists a wavelet $g \in \Sigma_{h}$ such that for all $u \in \Sigma_{h}$ we have

$$
\Phi[g] \leq \Phi[u]
$$

In other words, the localization functional $\Phi$ has a minimizer in each $\Sigma_{h}$, for all $h$, which have some regularity as expressed through $\left|\mathcal{W}_{h} h\right| \leq w$. Note that we have to require, that $\Phi[h]<\infty$. This ensures, that the set of functions having a $w$ localization is not empty. In turn, this is a requirement for $w$ in which it should not be decaying too fast (e.g. compactly supported weights are not possible).

Actually this kind of results can be generalized to a more abstract setting as follows: Consider two Banach spaces $B, K \subset L^{2}(G, d \lambda+d \rho)$ of functions over $G$ with continuous embeddings. $B$ should be a lattice, $\||s|\|_{B}=\|s\|_{B}$. We then can define a localization with respect to $B$ simply as

$$
\Phi[g]=\left\|\mathcal{W}_{g} g\right\|_{B}
$$

We include the value $\Phi=\infty$ in the natural way. For $B$ and $K$ we further suppose that the following holds:

Invariance of $B \quad B$ should be $G$ bi-invariant: for all $y \in G$ there is an $b>0$ such that

$$
\|s(y \circ \cdot)\|_{B} \leq b\|s\|_{B}, \quad\|s(\cdot \circ y)\|_{B} \leq b\|s\|_{B} .
$$

It should be stable under inversion

$$
\left\|s\left(x^{-1}\right)\right\|_{B} \leq d\|s\|_{B} .
$$

Then we have $u(x)=s\left(y^{-1} \circ x \circ y\right)$ satisfies $\|u\|_{B} \leq e\|s\|_{B}$.

Semi-continuity of $B$ norm Suppose further that the following inequality holds for the norm in $B$ : if $s_{n} \in B$ is any sequence of non-negative functions $s_{n} \geq 0$ then consider $s=\liminf _{n \rightarrow \infty} s_{n}$. Then we require that

$$
\|s\|_{B}=\left\|\liminf s_{n}\right\|_{B} \leq \liminf _{n \rightarrow \infty}\left\|s_{n}\right\|_{B} .
$$

In classical $L^{p}$ spaces this is a direct consequence of Fatou's lemma

$$
\int \liminf s_{n} d \mu \leq \liminf \int s_{n} d \mu \text {. }
$$

Compact embedding of $K$ For the space $K$ we suppose that the following compact embedding property holds: let $L \subset K$ be a $K$-bounded set. If now on each compact subset of $G$ the set of functions $L$ is uniformly continuous then $L$ is precompact in $L^{2}(G, d \lambda+d \rho)$.

Convolution mapping The two spaces $B$ and $K$ are linked through the following convolution property: for fixed $r \in B$ the convolution product with $r$ is a linear operator

$$
*_{r}: B \rightarrow K, \quad u \mapsto r * u,
$$

and it is bounded $\|r * u\|_{K} \leq d\|u\|_{B}$, with $d$ depending only on $r$. 
Nonempty We suppose that there is at least one $h \in D(T), h \neq 0$ such that $\Phi[h]<\infty$. This $h$ is admissible, $h \in A$, since $B \subset L^{2}(G, d \lambda+d \rho)$. and thanks to the following lemma.

Lemma 1 Let $h \in A, h \neq 0$ be given. Then $g \in H$ is actually in $A$ if and only if

$$
\|g\|^{2}=\left\|\mathcal{W}_{h} g\right\|_{L^{2}(G, d \lambda)}^{2}+\left\|\mathcal{W}_{h} g\right\|_{L^{2}(G, d \rho)}^{2}<\infty .
$$

The square root of the left hand side defines a norm which on $A$ is equivalent to the norm of $A$ :

$$
c_{h}^{-1}\left\|\mathcal{W}_{h} g\right\|_{L^{2}(G, d \lambda)}^{2}+\|h\|_{H}^{-2}\left\|\mathcal{W}_{h} g\right\|_{L^{2}(G, d \rho)}^{2}=\|g\|_{A}^{2} .
$$

Proof. Suppose $g \in A$. Thanks to the formula

$$
\left\|\mathcal{W}_{h} g\right\|_{L^{2}(G, \rho)}^{2}=\left\|\mathcal{W}_{g} h\right\|_{L^{2}(G, d \lambda)}^{2}=c_{g}\|h\|_{H}^{2}
$$

and the isometric property of the wavelet transform we may conclude.

Under the above conditions, the following theorem holds.

Theorem 2 There is a $g \in \Sigma_{h} \cap A$ such that for all $u \in \Sigma_{h}$ we have

$$
\Phi[g] \leq \Phi[u]
$$

This function $g$ then satisfies $\mathcal{W}_{h} g \in K$.

Typical examples for such spaces $B$ are as follows. As space $B$ we take the functions for which we have

$$
\|r\|_{w, \infty}=\left\|w^{-1} r\right\|_{L^{\infty}(G)}
$$

We write also $B_{w, \infty}$ for this space. For the space $K$ we take the analogue space of functions with weight $u=w * w$. If now $u \in L^{2}(G, d \lambda+d \rho)$ then we are in the setting of the first theorem. We only have to convince ourselves that all properties of the stated spaces are satisfies. The only non-trivial property is now the compact embedding.

Lemma 2 Let $u>0$ be a symmetric weight function with $u \in L^{2}(G, d \lambda+d \rho)$. Then any set of functions which are uniformly continuous on compact sets of $G$ is precompact in $L^{2}(G, d \lambda)$.

Proof. Let $s_{n}$ be a sequence of functions in this set of functions. Since $G$ is $\sigma$ compact we can find a sequence of compact sets $K_{m} \subset G$ with $K_{m} \subset K_{m+1}$ and $\bigcup K_{m}=G$. Upon choosing a subsequence we may require that

$$
\int_{G \backslash K_{m}} u^{2}(d \lambda+d \rho) \leq 1 / m
$$

On each $K_{m}$ we can therefore find a uniformly convergent subsequence. Therefore upon choosing a suitable diagonal subsequence we may suppose that for $m^{\prime}>m$

$$
\int_{K_{m}}\left|s_{m}-s_{m^{\prime}}\right|^{2}(d \lambda+d \rho) \leq 1 / m
$$


We therefore have

$$
\begin{aligned}
\left\|s_{m}-s_{m^{\prime}}\right\|_{L^{2}(G, d \lambda+d \rho)}^{2}= & \int_{G \backslash K_{m}}\left|s_{m}-s_{m^{\prime}}\right|^{2}(d \lambda+d \rho) \\
& +\int_{K_{m}}\left|s_{m}-s_{m^{\prime}}\right|^{2}(d \lambda+d \rho) \leq 2 / m
\end{aligned}
$$

and thus $s_{m}$ is a Cauchy sequence. Its pointwise limit exists, and thanks to Fatou lemma is in $L^{2}(G, d \lambda+d \rho)$.

A second family is given by

$$
\|r\|_{w, 2}=\left\|w^{-1 / 2} r\right\|_{L^{2}(d \lambda+d \rho)} .
$$

We denote by and $B_{w, 2}$ the associated Banach space. This means, we consider localization quantities of the form

$$
\int_{G} w^{-1}\left|\mathcal{W}_{g} g\right|^{2} d \lambda
$$

If $w$ is such that

$$
\eta(x)=\sup _{y \in G} \sqrt{w\left(y^{-1} \circ x\right) w(y)}
$$

satisfies $\eta \in L^{2}(G, d \lambda+d \rho)$, we may estimate for $r=w^{1 / 2} u, s=w^{1 / 2} v, u, v \in L^{2}(G, d \lambda+$ $d \rho)$ that

$$
\begin{aligned}
|r * s(x)| & \leq \int w\left(y^{-1} \circ x\right) w(y) u\left(y^{-1} \circ x\right) v(y) d \lambda(y) \mid \\
& \leq \eta(x)\|u\|_{L^{2}(G, d \rho)}\|v\|_{L^{2}(G, d \lambda)} \leq \eta(x)\|r\|_{w, 2}\|s\|_{w, 2}
\end{aligned}
$$

and thus we have $B_{w, 2} * B_{w, 2} \subset B_{\eta, \infty}$. Therefore the theorem applies if we choose for the space $K=B_{\eta, \infty}$. and we have the existence of an optimally localized reconstruction wavelet.

We now prove the theorem. To start we analyze the mapping properties of $\Phi$. We denote by $\Delta$ the domain of $\Phi$.

$$
\Delta=\{s \in H: \Phi[s]<\infty\} \subset A .
$$

Lemma 3 The functional $\Phi$ is strongly $H$ - lower semi-continuous on $\Delta$. More precisely for $\Delta \ni u_{n} \rightarrow u \in H$ in $H$, we have

$$
\Phi[u] \leq \liminf _{n \rightarrow \infty} \Phi\left[u_{n}\right] .
$$

Proof. Since $\mathcal{W}_{u_{n}} u_{n} \rightarrow \mathcal{W}_{u} u$ point-wise and thus by hypothesis of semi-continuity of the $B$-norm

$$
\Phi\left[\lim _{n \rightarrow \infty} u_{n}\right]=\left\|\lim _{n \rightarrow \infty} \mathcal{W}_{u_{n}} u_{n}\right\|_{B} \leq \liminf _{n \rightarrow \infty}\left\|\mathcal{W}_{u_{n}} u_{n}\right\|=\liminf _{n \rightarrow \infty} \Phi\left[u_{n}\right]
$$

We even have 
Lemma 4 The functional $\Phi$ is $H$-weakly lower semi-continuous on $\Delta \backslash\{0\}$. More precisely, for any $H$-weak convergent sequence $\Delta \ni g_{n} \rightarrow g \in H, g \neq 0$ with $g_{n} \in \Delta$ we have $\Phi[g] \leq \lim \inf \Phi\left[g_{n}\right]$

Proof. Let

$$
\gamma=\liminf _{n \rightarrow \infty} \Phi\left[g_{n}\right] .
$$

Clearly $\gamma \geq 0$. In the case that $\gamma=\infty$ the lemma holds true and we may suppose $0 \leq \gamma<\infty$. We may find a subsequence which denote by $g_{n}$ with $\Phi\left[g_{n}\right]<\infty$, and $\Phi\left[g_{n}\right] \rightarrow \gamma$. By hypothesis there is an $h \in D(T)$ with $\Phi[h]<\infty$. By the invariance of $B$ it follows thanks to

$$
\left(\mathcal{W}_{U(y) h} U(y) h\right)(x)=\mathcal{W}_{h} h\left(y^{-1} \circ x \circ y\right)
$$

that the whole orbit of $h$ has the same properties. Since the representation is irreducible and $T$ has dense range, we may suppose that $c_{g, h}=(g, T h)_{H} \neq 0$. For $s \in A$ we have by continuity of the convolution

$$
\left\|\mathcal{W}_{h} s\right\|_{K}=\left\|\Pi * \mathcal{W}_{s} s\right\|_{K} \leq d\left\|\mathcal{W}_{s} s\right\|_{B}=d \Phi[s], \quad \Pi=c_{s, h}^{-1} \mathcal{W}_{h} h .
$$

By weak convergence we have $c_{g_{n}, h}=\left(g_{n}, T h\right)_{H} \rightarrow(g, T h)_{H} \neq 0$. Therefore, since $g_{n} \in A$ tanks to $\Phi\left[g_{n}\right]<\infty$, we may conclude by setting $s=g_{n}$ in the formula above that $\left\{\mathcal{W}_{h} g_{n}\right\}$ is a bounded set in $K$ and hence it is bounded in $\mathcal{W}_{h} H$ (the image of $H$ under $\mathcal{W}_{h}$ ) too. Since the representation of the wavelet transform is strongly continuous this family of functions is uniformly continuous on any compact subset of $G$. Because of the compact embedding property of $K$ we can extract an $H$ convergent subsequence $g_{m(n)} \rightarrow g$. Since $\Phi$ is strongly lower continuous we have $\Phi[g] \leq \lim \inf \Phi\left[g_{n}\right]=\gamma$.

Now the proof of the main theorem is easy.

Proof. Let $\Phi\left[g_{n}\right] \rightarrow \gamma=\inf _{g \in \Sigma_{h}} \Phi[g]$. Since as before $\Phi\left[g_{n}\right] \leq b\left\|g_{n}\right\|_{H}$ we see that $g_{n}$ is bounded in $H$. Thanks to the Banach-Alaoglou theorem we may extract an $H$ - weakly convergent subsequence $g_{m(n)} \rightarrow g$ weakly. Since $\Phi$ is weekly lower semi-continuous we may conclude that $\Phi[g]=\gamma$. The set $\Sigma_{h}$ is weekly closed and hence $g \in \Sigma_{h}$.

We can even prove the following optimal localization result.

Theorem 3 There is $g$ with $\|g\|_{H}=1$, such that for all $u \in H$, with $\|u\|_{H}=1$ we have

$$
\Phi[g] \leq \Phi[u] .
$$

Proof. As before, we find a weekly convergent sequence $g_{n} \rightarrow g$ weekly with $\Phi[g]=$ $\inf _{\|s\|=1} \Phi[s]$. Now as in the proof of lemma 4 we see that there is a strongly convergent subsequence $g_{m(n)} \rightarrow g$ and thus $\|g\|=1$. 


\section{The numerical approximation of localized states}

As we have shown in the previous section, for each weight function satisfying certain conditions, there exists an optimally localized wavelet $g$. The term 'optimality' is strongly connected with the associated Banach space norm $\|\cdot\|_{B}$.

Before providing a receipt of how to derive those optimally localized wavelets, we wish to be more concrete and to give a few examples of typical Banach spaces and weight functions to which our theory can be applied. To this end, let us consider the class of $\alpha$-modulation spaces which are usually defined by means of the flexible Gabor-wavelet transform, see [3],

$$
\mathcal{W}_{g}^{\alpha}(f)(x)=\left(f, U\left(\sigma_{\alpha}(x)\right) g\right)_{H} .
$$

Since this transform is based on square integrability modulo quotients, we limit the subsequent consideration to the cases $\alpha=0$ and $\alpha=1$ (which fits then quite nicely with our framework, see below). For $\alpha=0$, the family $\{U(\sigma(x)) g\}$ is a Gabor system and $\mathcal{W}_{g}^{0} f$ coincides with the classical short time Fourier transform, while for $\alpha \rightarrow 1$ the family tends to the situation encountered in the wavelet context, where $\mathcal{W}_{g}^{1}$ is just a slight modification of the continuous wavelet transform. The intermediate case $\alpha=1 / 2$ appears in the literature as the Fourier-Bros-Iagolnitzer transform [2, 14]. In particular, one characterizes $\alpha$-modulation spaces as follows (for simplicity we consider the wavelet transform of functions over $\mathbb{R})$ : for $s \in \mathbb{R}$, for all $1 \leq p, q \leq \infty$, and for $\alpha \in[0,1)$

$$
M_{p, q}^{s+\alpha(1 / q-1 / 2), \alpha}(\mathbb{R})=\left\{f \in \mathcal{S}^{\prime}: \mathcal{W}_{g}^{\alpha}(f) \in L^{p, q, s}\right\}, \quad\|f\|_{M_{p, q}^{s+\alpha(1 / q-1 / 2), \alpha}} \asymp\left\|\mathcal{W}_{g}^{\alpha}(f)\right\|_{L^{p, q, s}},
$$

where $L^{p, q, s}\left(\mathbb{R}^{2}\right)$ is the space of functions $F$ on $\mathbb{R}^{2}$ such that

$$
\|F\|_{L^{p, q, s}}:=\left(\int_{\mathbb{R}}\left(\int_{\mathbb{R}}|F(t, \omega)|^{p} d t\right)^{q / p}(1+|\omega|)^{s q} d \omega\right)^{1 / q}<\infty .
$$

For $\alpha=0$, the space $M_{p, q}^{s, 0}(\mathbb{R})$ coincides with the modulation space $M_{p, q}^{s}(\mathbb{R})$. For $\alpha \rightarrow 1$ the space $M_{p, q}^{s, 1}(\mathbb{R})$ coincides with the inhomogeneous Besov space $B_{p, q}^{s}(\mathbb{R})$. These spaces serve as a reservoir of smoothness spaces in which functions can be characterizes by means of special analyzing atoms or a so-called frame. A desirable property of the analyzing atom is localization with respect to underlying Banach spaces metric, i.e. in this context an optimal localized $g$ is associated with Banach space norm $\|\cdot\|_{M_{p, q}^{s+\alpha(1 / q-1 / 2), \alpha}}$ which in turn is here characterized by the weight

$$
w(\omega)=(1+|\omega|)^{s} .
$$

The numerical scheme developed below is limited to case $p=q=2$ and applies thus not to all situations.

Let us now consider a concrete case which is a little beyond the above mentioned examples. Let $L^{2, w^{-1}}$ with symmetric weight function

$$
w^{-1}(x)=w^{-1}(a, b)=\left(|a|+|a|^{-1}\right)^{4} \cdot\left(1+|b|(1+|a|)^{-1}\right)^{4}
$$


be the space under consideration. Then, in accordance with Theorem 3, the optimization problem can be casted as follows

$$
\Phi(g)=\left\|\mathcal{W}_{g} g\right\|_{L^{2, w^{-1}}}^{2}+\alpha\|g\|_{H}^{2} .
$$

In order to discretize the problem somehow, we may represent $g$ by means of some frame $\left\{\psi_{\lambda}\right\}_{\lambda \in \Lambda} \subset H$, i.e.

$$
g=\sum_{\lambda \in \Lambda} g_{\lambda} \psi_{\lambda}
$$

Consequently, the goal is to reconstruct a sequence $\left\{g_{\lambda}\right\}_{\lambda \in \Lambda}=\mathrm{g} \in \ell_{2}$ for which $\Phi(g) \leq$ $\Phi(s)$, for all $s \in H$.

Introducing for some $x \in G$ the infinite matrix $\mathbf{A}(x)=\left(\left(\psi_{\lambda}, U(x) \psi_{\eta}\right)_{H}\right)_{\lambda, \eta \in \Lambda}$, the wavelet transform reads as $\mathcal{W}_{g} g(x)=\langle\mathbf{g}, \mathbf{A}(x) \mathbf{g}\rangle_{\ell_{2}}=: F[\mathbf{g}](x)$. Obviously, $F[\mathbf{g}](e)=$ $\|g\|_{H}^{2}$, and thus we may write

$$
\Phi(\mathbf{g})=\|F[\mathbf{g}]\|_{L^{2, w^{-1}}}^{2}+\alpha F[\mathbf{g}](e) .
$$

Since the optimization problem is no longer convex, we have to apply adequate strategies for nonlinear problems. We suggest to make use of a Tikhonov-based iteration method for nonlinear problems which was developed in [16]. The technology to be applied here will always find a critical point of $\Phi$, and under additional assumptions on $F$ and the solution one can assure that the critical point is a global minimizer.

The method borrowed from [16] goes now as follows: Firstly, in order to obtain a problem which is hopefully easier to solve, we replace $\Phi$ by

$$
\Phi^{s}(\mathbf{g} ; \mathbf{a}):=\Phi(\mathbf{g})+C\|\mathbf{g}-\mathbf{a}\|_{\ell_{2}}^{2}-\|F[\mathbf{g}]-F[\mathbf{a}]\|_{L^{2, w^{-1}}}^{2},
$$

where $\mathbf{a}$ is some auxiliary element in $\ell_{2}$. So far its not clear whether $\Phi^{s}$ is positive or even bounded from below. Following the lines in [16], i.e. choosing for $\alpha>0$ a ball around the origin $K_{r}$ and $C$ adequately large (in dependence on $F$ and $\Phi(\mathbf{a})$ ), one can assure for all $\mathrm{g} \in K_{r}, \Phi(\mathbf{g}) \leq \Phi^{s}(\mathbf{g} ; \mathbf{a})$.

The iteration process is now obtained by picking some initial $\mathbf{g}_{0}=\mathbf{a}$ and therewith some proper $C>0$ and by deriving a sequence $\left\{\mathbf{g}_{k}\right\}_{k \in \mathbf{N}}$ via

$$
\mathbf{g}_{k+1}=\arg \min _{\mathbf{g}} \Phi^{s}\left(\mathbf{g} ; \mathbf{g}_{k}\right) \text {. }
$$

From this iteration we expect convergence at least towards a critical point of $\Phi$. First, we have to make sure that the sequence of functionals is properly defined:

Lemma 5 Let $\mathbf{a}$ be given and $K_{r}, C$ be defined as in [16]. Then for all $k \in \mathbf{N}, \Phi^{s}\left(\mathbf{g} ; \mathbf{g}_{k}\right)$ are bounded from below, and, moreover, for the minimizers $\mathbf{g}_{k+1}$ holds $\mathbf{g}_{k+1} \in K_{r}$.

Let now $\mathbf{A}$ be the shorthand for $\mathbf{A}(e)$. A simple calculation shows:

Lemma 6 The necessary condition for a minimum of (4.1) reads as

$$
\mathbf{g}=\frac{1}{C}\left\{\mathbf{g}_{k}-\alpha \mathbf{A g}-F^{\prime}[\mathbf{g}]^{*}\left(F\left[\mathbf{g}_{\mathbf{k}}\right] w^{-1}\right)\right\}
$$




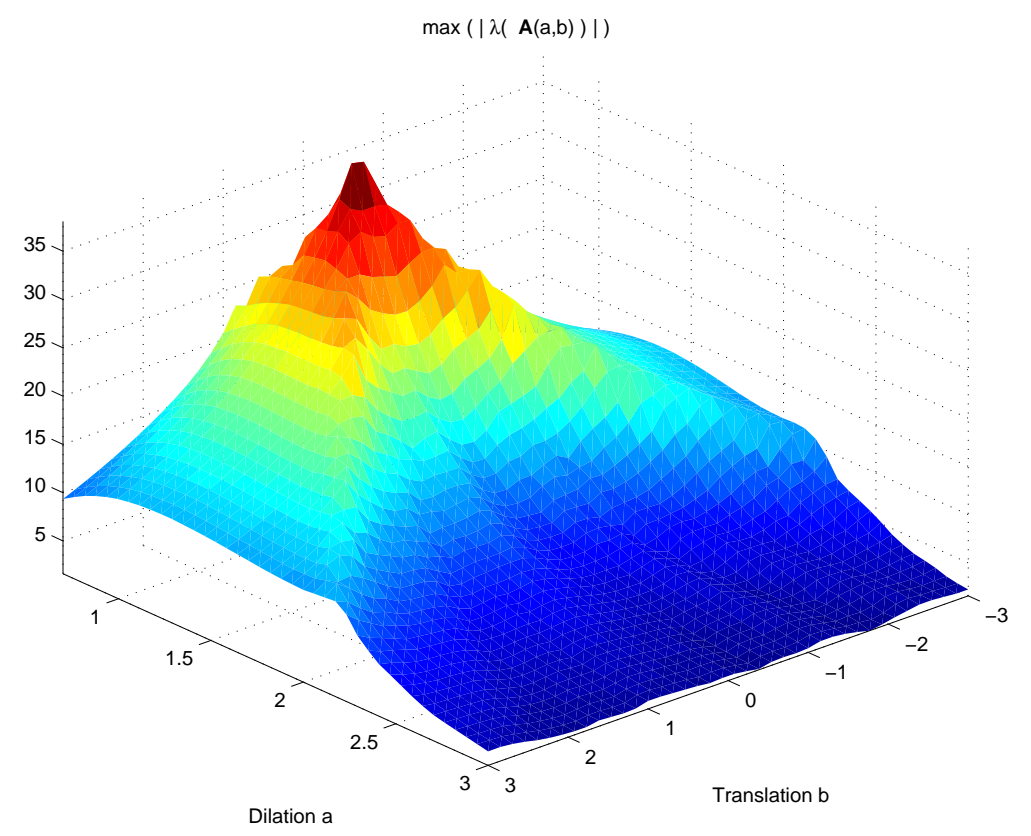

Figure 1: Maximal Eigenvalues of the infinite matrices $A(a, b)$ for all the $(a, b) \in G$ used in the frame representation.

The hope is that the right hand side of (4.2) defines a contraction. A straightforward computation shows,

$$
\left\|\mathbf{g}-\mathbf{g}^{\prime}\right\|_{\ell_{2}} \leq \frac{1}{C}\left\{\alpha|\|\mathbf{A}\||+2\|\| \mathbf{A}(\cdot)\|\|\left\|_{L^{2, w^{-1}}}\right\| F\left[\mathbf{g}_{k}\right] \|_{L^{2, w^{-1}}}\right\}\left\|\mathbf{g}-\mathbf{g}^{\prime}\right\|_{\ell_{2}} .
$$

To bound this quantity requires the Lipschitz-continuity of $F^{\prime}[\mathbf{g}]$, or in other words, the finiteness of \|\| $\mathbf{A}(\cdot)\left|\|\mid\|_{L^{2, w^{-1}}}\right.$ which is difficult to prove, but can be verified numerically: we may consider the spectral radius $\rho(\mathbf{A}(a, b))$ (for a particular frame, see below) as a function of $(a, b) \in G$. Figure 1 shows a sufficient decay of $\rho(\mathbf{A}(a, b))$ and assures therewith that, for $C$ large enough, the convergence of the fixed point iteration (4.2) towards a unique minimizer $\mathbf{g}_{k+1}$ of $\Phi^{s}\left(\mathbf{g} ; \mathbf{g}_{k}\right)$ can be achieved. Moreover, we have with the help of [16] that the sequence $\left\{\mathbf{g}_{k}\right\}$ converges at least towards a critical point of $\Phi$. If we could impose more smoothness on $F$ and on the solution $\mathrm{g}$ to be reconstructed, we could also achieve uniqueness.

Next, we have to ensure that $\left\|g^{n+1}\right\|_{H}^{2}=1$ (the index $n$ stands now for the fixed point iteration) holds true through the whole fixed point iteration process, i.e. we have to determine $\alpha$ in each iteration step:

$$
\begin{aligned}
\left(\mathbf{g}^{n+1}, \mathbf{A g}^{n+1}\right)_{\ell_{2}}=\frac{1}{C^{2}}\{ & \alpha^{2} F\left[\mathbf{A g}^{n}\right](e)- \\
& 2 \alpha \Re\left(\mathbf{A g}^{n}, \mathbf{A}\left(\mathbf{g}_{k}-F^{\prime}\left[\mathbf{g}^{n}\right]^{*}\left(F\left[\mathbf{g}_{\mathbf{k}}\right] w^{-1}\right)\right)\right)_{\ell_{2}} \\
& \left.+F\left[\mathbf{g}_{k}-F^{\prime}\left[\mathbf{g}^{n}\right]^{*}\left(F\left[\mathbf{g}_{k}\right] w^{-1}\right)\right](e)\right\},
\end{aligned}
$$

i.e. finding $\alpha=\alpha^{n+1}$ amounts to finding the roots of a real parabola. With the shorthand 

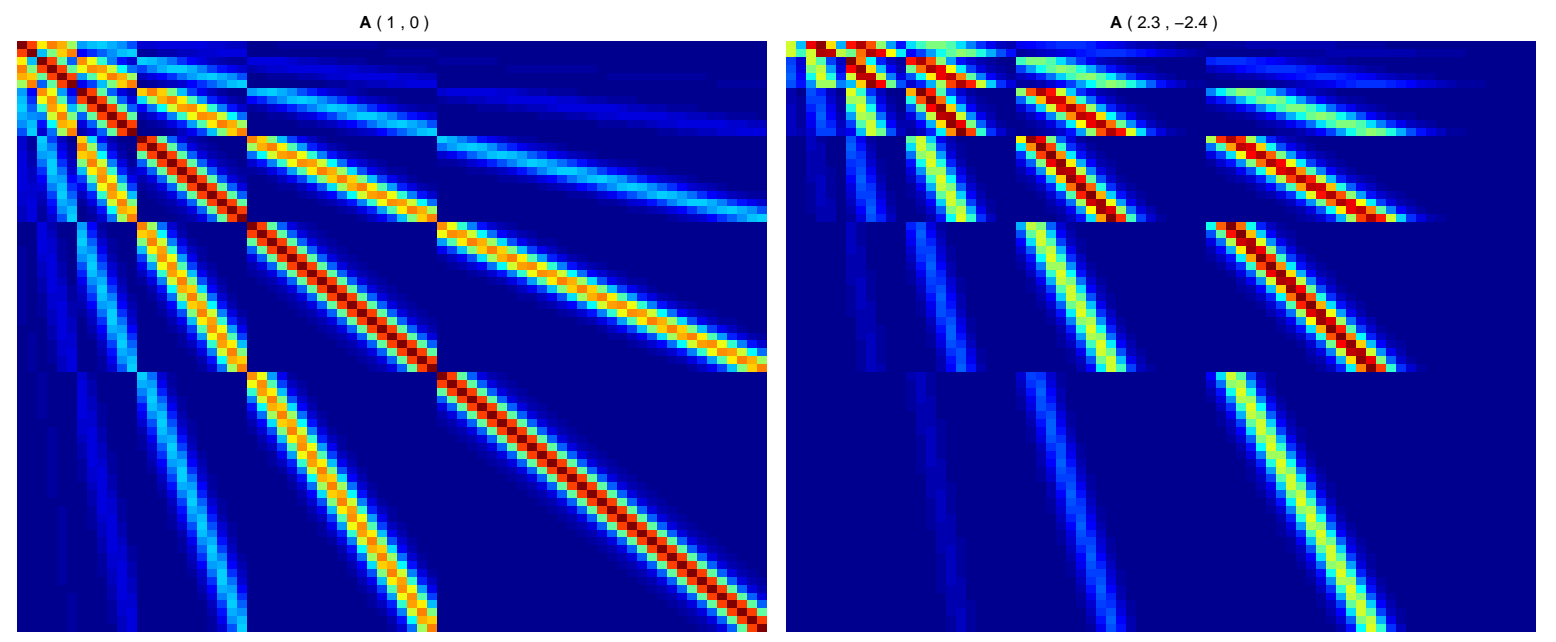

Figure 2: Structure of $A(a, b)$ for two particular cases; left: $(a, b)=(1,0)$ and right $(a, b)=(2.3,-2.4)$.

$$
\begin{aligned}
M= & \Re\left(\mathbf{A g}^{n}, \mathbf{A}\left(\mathbf{g}_{k}-F^{\prime}\left[\mathbf{g}^{n}\right]^{*}\left(F\left[\mathbf{g}_{\mathbf{k}}\right] w^{-1}\right)\right)\right)_{\ell_{2}}, \text { we obtain } \\
& \alpha^{n+1}=\frac{M \pm\left(M^{2}-F\left[\mathbf{A g}^{n}\right](e)\left\{F\left[\mathbf{g}_{k}-F^{\prime}\left[\mathbf{g}^{n}\right]^{*}\left(F\left[\mathbf{g}_{k}\right] w^{-1}\right)\right](e)-C^{2}\right\}\right)^{1 / 2}}{F\left[\mathbf{A} \mathbf{g}^{n}\right](e)}
\end{aligned}
$$

Now we can summarize an algorithm for computing a critical sequence $\mathbf{g}$ for the minimization problem $\inf _{\|g\|_{H}=1} \Phi[g]$ :

- pick some initial $\mathbf{g}_{0}$ (not too far off the expected solution) and some $C>0$ (large enough)

- compute $\mathbf{g}_{k+1}=\arg \min _{\mathbf{g}} \Phi^{s}\left(\mathbf{g} ; \mathbf{g}_{k}\right)$ via fixed point iteration (4.2):

$$
\begin{aligned}
& \text { - compute } \alpha^{n+1}=\max \left\{\alpha_{1}^{n+1}, \alpha_{2}^{n+1}\right\} \text { via (4.3) } \\
& \text { - compute } \mathbf{g}^{n+1} \text { via }(4.2) \\
& \text { - } \mathbf{g}_{k+1}=\lim _{n \rightarrow \infty} \mathbf{g}^{n+1}
\end{aligned}
$$

In what follows we aim to illustrate the computation of an optimally localized wavelet. For sake of simply computing the operators $\mathbf{A}(x)$, we have chosen a (finite dimensional) Cauchy wavelet frame $\left\{\psi_{\lambda}\right\} \subset L^{2}(\mathbf{R})$ of order $N$ (here $\mathrm{N}=3$ ). Thus, $\mathbf{A}(x)$ can be derived for each $x \in G$ explicitly, see Figure 2. The resulting iteration process to reconstruct at least a critical $g$ is illustrated in Figure 3, and the final approximation with the time representation in Figure 4.

\section{References}

[1] B. Borden. Mathematical problems in radar inverse scattering. Inverse Problems, 18(1):R1-R28, 2002. 

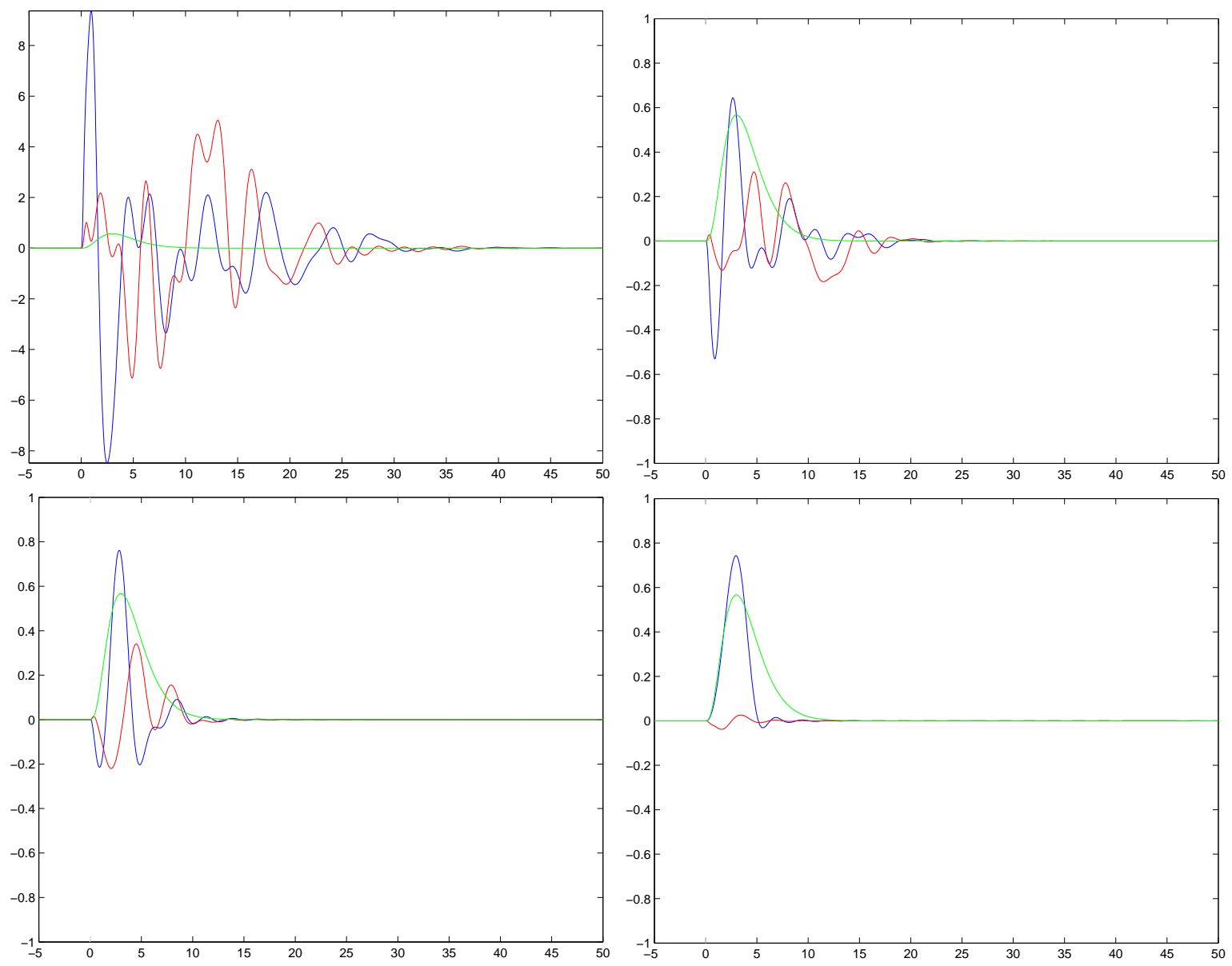

Figure 3: From top left to up right: Fourier representations of initial $g_{0}$ (not normalized), $g_{4}, g_{10}$, and $g_{30}$ (blue/red - real and imaginary part; green - Cauchy wavelet).
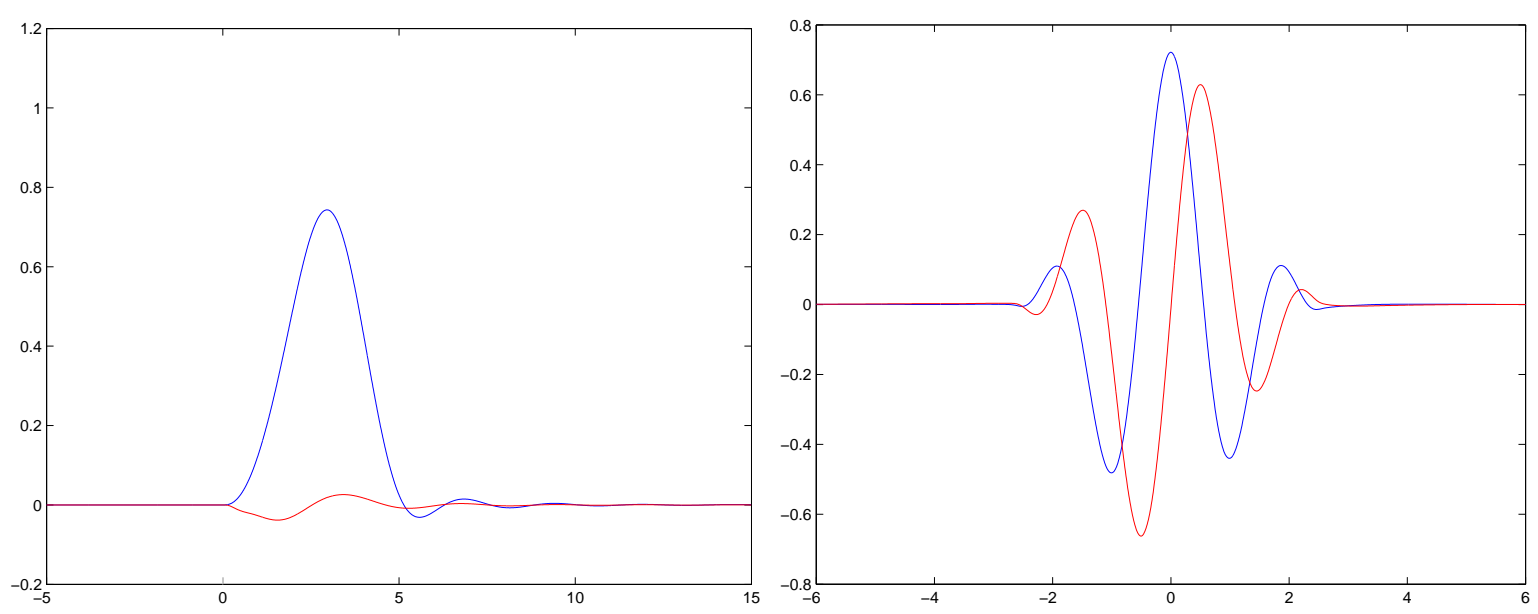

Figure 4: Left: Fourier representation of the approximated optimally localized coherent state; right: associated time representation. 
[2] J. Bros and D. Iagolnitzer. Support essentiel et structure analytique des distributions. Seminaire Goulaouic-Lions-Schwartz, 18, 1975.

[3] S. Dahlke, M. Fornasier, H. Rauhut, G. Steidl, and G. Teschke. Generalized Coorbit Spaces, Banach Frames and Relations to $\alpha$-Modulation Spaces. Preprint, 2005.

[4] S. Dahlke, V. Lehmann, and G. Teschke. Applications of Wavelet Methods to the Analysis of Meteorological Radar Data - An Overview. (invited paper) Arabian Journal of Science and Engineering, 28(1C):3-44, 2003.

[5] S. Dahlke and P. Maaßs. The affine uncertainty principle in one and two dimensions. Comp. Math. Appl., 30(3-6):293-305, 1995.

[6] S. Dahlke, P. Maass, and G. Teschke. Reconstruction of Wideband Reflectivity Densities by Wavelet Transforms. Advances in Computational Mathematics, 18(24):189-209, 2003.

[7] S. Dahlke, P. Maass, and G. Teschke. Reconstruction of Reflectivity Densities in a Narrowband Regime. IEEE Transactions on Antennas and Propagation, 52(6):16031606, 2004.

[8] I. Daubechies. Time-frequency localisation operators: A geometric phase space approach. IEEE Transactions on Information Theory, 34:605-612, 1988.

[9] I. Daubechies and Th. Paul. Time-frequency localisation operators-a geometric phase space approach: II the use of dilations. Inverse Problems, 4:661-680, 1988.

[10] M. Duflo and C. C. Moore. On the regular representation of a nonunimodular locally compact group. J. Funct. Anal., 21:209-243, 1976.

[11] A. Grossmann, J. Morlet, and T. Paul. Transforms associated to squareintegrable group representations i. Math. Phys., 27, 1985.

[12] A. Grossmann, J. Morlet, and T. Paul. Transforms associated to squareintegrable group representations ii. Ann. Inst. H. Poincaré, 45, 1986.

[13] M. Holschneider. Wavelets An Analysis Tool. Clarendon Press, Oxford, 1995.

[14] M. Holschneider and B. Nazaret. An interpolation family between Gabor and wavelet transformations. Application to differential calculus and construction of anisotropic Banach spaces. in: Advances in Partial Differential Equations, Albeverio, Demuth, Schrohe, Schulze (eds.), Wiley:363-394, 2003.

[15] H. J. Landau and H. O. Pollak. Prolate spheroidal wave functions, fourier analysis and uncertainty, II. Bell Syst. Tech. J., 40:65-84, 1961.

[16] R. Ramlau and G. Teschke. Tikhonov Replacement Functionals for Iteratively Solving Nonlinear Operator Equations. Inverse Problems, 21:1571-1592, 2005. 
[17] D. Slepian and H. O. Pollak. Prolate spheroidal wave functions, fourier analysis and uncertainty, I. Bell Syst. Tech. J., 40:43-64, 1961.

[18] G. Teschke. Construction of Generalized Uncertainty Principles and Wavelets in Bessel Potential Spaces. International Journal of Wavelets, Multiresolution and Information Processing, 3(2), 2005. 